\title{
Immunization for Ebola virus infection
}

\author{
Ling $\mathrm{XU}^{1,2}{ }^{1,}$ Anthony SAnchez ${ }^{3}$, Zhi-Yong YanG ${ }^{1,4}$, Sherif R. Zaki ${ }^{3}$, Elizabeth G. NAbel ${ }^{2}$, \\ STUART T. NiCHOL ${ }^{3} \&$ GARY J. NABEL ${ }^{1,2,4}$

\begin{abstract}
'Department of Biological Chemistry, ${ }^{2}$ Department of Internal Medicine and ${ }^{4}$ Howard Hughes Medical Institute, University of Michigan Medical Center, 1150 West Medical Center Drive, 4520 MSRB I, Ann Arbor, Michigan 48109-0650, USA

${ }^{3}$ Special Pathogens Branch, Division of Viral and Rickettsial Diseases, National Center for Infectious Diseases, Centers for Disease Control and Prevention, 1600 Clifton Road, Atlanta, Georgia 30333, USA

Correspondence should be addressed to G.J.N.
\end{abstract}

\begin{abstract}
Infection by Ebola virus causes rapidly progressive, often fatal, symptoms of fever, hemorrhage and hypotension. Previous attempts to elicit protective immunity for this disease have not met with success. We report here that protection against the lethal effects of Ebola virus can be achieved in an animal model by immunizing with plasmids encoding viral proteins. We analyzed immune responses to the viral nucleoprotein (NP) and the secreted or transmembrane forms of the glycoprotein (sGP or GP) and their ability to protect against infection in a guinea pig infection model analogous to the human disease. Protection was achieved and correlated with antibody titer and antigen-specific T-cell responses to SGP or GP. Immunity to Ebola virus can therefore be developed through genetic vaccination and may facilitate efforts to limit the spread of this disease.
\end{abstract}

The Ebola viruses and the genetically related Marburg virus are filoviruses associated with outbreaks of highly lethal hemorrhagic fever in humans and primates in North America, Europe and Africa $^{1,2}$. Ebola viruses are negative-stranded RNA viruses comprised of four subtypes, including those described in the Zaire, Sudan, Reston and Ivory Coast episodes ${ }^{3}$. Although several subtypes have been defined, the genetic organization of these viruses is similar, each containing seven linearly arrayed genes. Among the viral proteins, the envelope glycoprotein exists in two alternative forms, a 50- to 70-kilodalton ( $\mathrm{kDa}$ ) secreted protein of unknown function encoded by the viral genome and a 130-kDa transmembrane glycoprotein generated by RNA editing that mediates viral entry ${ }^{1,3}$. Other structural gene products in-

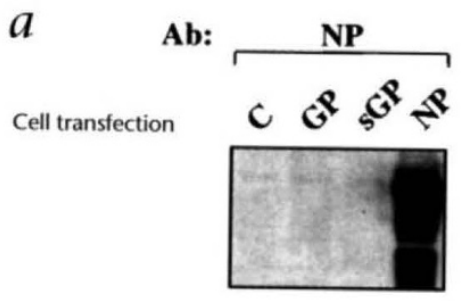

$\begin{array}{llll}1 & 2 & 3 & 4\end{array}$

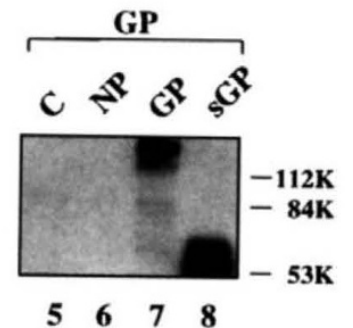

$5 \quad 6 \quad 7 \quad 8$ $b$

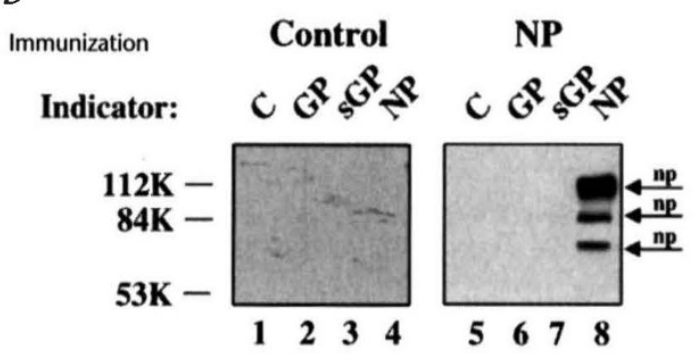

clude the nucleoprotein (NP), matrix proteins VP24 and VP40, presumed nonstructural proteins VP30 and VP35, and the viral polymerase (reviewed in ref. 1). Although spontaneous variation of its RNA sequence does occur in nature, there appears to be less nucleotide polymorphism within Ebola subtypes than among other RNA viruses ${ }^{3}$, suggesting that immunization may be useful in protecting against this disease.

Following the initial report that injection of plasmid DNA into muscle could direct the synthesis of recombinant proteins ${ }^{4}$, the suggestion was made that this gene transfer approach may be useful for vaccination and was termed genetic immunization ${ }^{5}$. This approach has been applied to different infectious diseases, including influenza ${ }^{6,7}$, malaria ${ }^{8,9}$ and tuberculosis ${ }^{10}$ and has also
Fig. 1 Expression of Ebola virus gene products in eukaryotic plasmid expression vectors. a, Expression vectors encoding the indicated viral gene products under regulation of the CMV immediate-early region 1 enhancer and promoter were prepared and transfected into 293 cells as previously described ${ }^{17,34}$. Cell extracts were prepared and analyzed by western blot analysis for NP (left) or GP (right) by using relevant rabbit antiserum and a secondary antibody, horseradish peroxidase-conjugated donkey anti-rabbit $\mathrm{lgG}$, at a dilution of 1:5000. Incubation with primary antibody was for $30 \mathrm{~min}$ at room temperature, and for $30 \mathrm{~min}$ at room temperature with secondary antibody. Immunocomplexes were then detected by chemiluminescence using super signal substrate reagents according to manufacturer's instructions. $\boldsymbol{b}$, Generation of antibody response in mice immunized with the indicated vectors and analyzed by western blot for NP, GP and SGP as shown. Antiserum from mice were tested at a dilution of 1:500 (NP), 1:50 (GP) or 1:50 (sGP), respectively, and developed with a secondary antibody (sheep anti-mouse, 1:5000) and chemiluminescence as in $a$. The control vector used for immunization represents the expression vector plasmid with no insert ${ }^{17}$. 
$a$ $\begin{array}{rc}\text { Immunization: } & \text { Control } \\ \text { Target: } & \text { NP }\end{array}$ NP
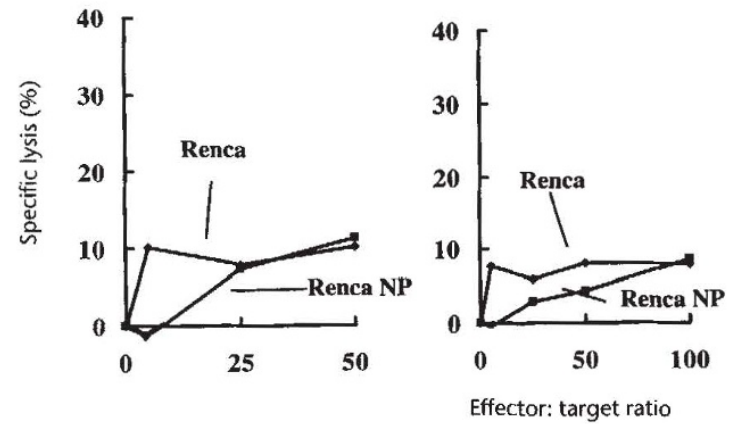

$b$
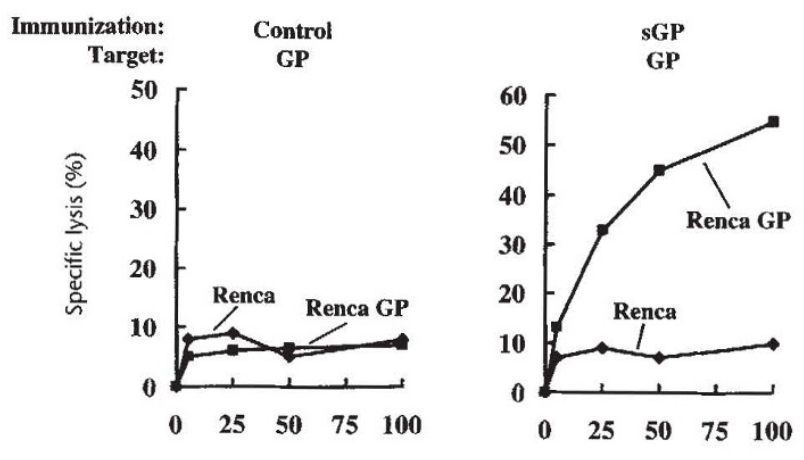

Effector: target ratio

C
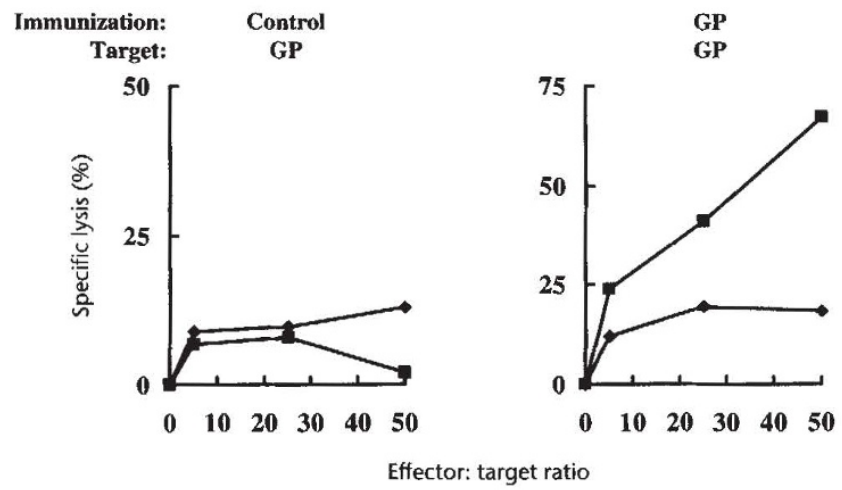

been used to modulate antibody and cell-mediated immune responses in autoimmune and allergic diseases ${ }^{11-14}$. Previous attempts to elicit protective immune responses against Ebola virus by using traditional active and passive immunization approaches have not succeeded ${ }^{1,15,16}$. We therefore sought to determine whether a genetic immunization approach might be useful in generating protection to this otherwise poorly immunogenic virus.

\section{Mouse immune responses to Ebola virus proteins}

,To characterize immune responses to selected Ebola virus proteins, eukaryotic expression vector plasmids were injected into mice. The cytomegalovirus (CMV) immediate early region 1 enhancer was used to stimulate transcription because it directs high levels of gene expression in muscle ${ }^{17}$. cDNAs encoding an abundant structural protein, the major viral nucleocapsid phospho-

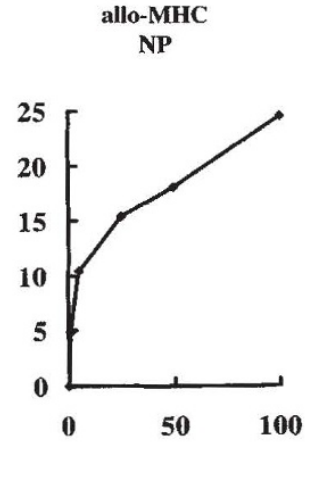

Fig. 2 Immune responses to NP and GP after genetic immunization in mice. $\boldsymbol{a}$, Splenic lymphocytes from vector- or NP-plasmid immunized mice were isolated approximately 6 weeks after the initial immunization and sensitized in vitro for 5 days with $10 \mathrm{U} / \mathrm{ml}$ hIL-2. Renca-NP cells sensitized splenocytes from vector-immunized or pCMV-NP immunized mice were used to detect CTL activity at the indicated effector:target (E/T) ratios on Renca or Renca-NP cells (left, middle) or with allogeneic effector cells with Renca-NP to show that they are susceptible to lysis (right). Allogeneic effector cells were generated by incubating cells derived from mice with a C57BL/6 background $\left(5 \times 10^{6} / \mathrm{ml}\right)$ with irradiated BALB/C spleen cells $\left(5 \times 10^{6} / \mathrm{ml}\right)$ in the presence of IL-2 $(20 \mathrm{U} / \mathrm{ml})$ for 5 days. The chromium release $\mathrm{CTL}$ assay with Renca-NP cells was performed in triplicate as previously described ${ }^{35}$. b, BALB/c female mice were immunized with the SGP plasmid expression vector and analyzed for their ability to lyse the syngeneic Renca cell line stably expressing GP. Isolation of stable transfectants, confirmation of expression, and CTL assay were performed as described (see the Methods section). Renca-GP- or SGPsensitized splenocytes from pCMV-sGP $(b)$ or pCMV-GP (c) immunized mice were used to determine the specific killing of ${ }^{51} \mathrm{Cr}$-labeled Renca-GP cells at the indicated E/T ratios. c, Mice immunized with GP were analyzed for their ability to lyse a syngeneic CT26 cell stably expressing GP or a CT26 vector control transduced line at the indicated E/T ratios. $\boldsymbol{d}$, Cellular proliferative response in the indicated immunized mice. $T$ cells, enriched or depleted (see Methods), were incubated at $10^{5}$ cells $/ \mathrm{ml}$ with $\mathrm{sGP}$-conditioned medium (25\%). Background was determined with cells incubated in medium from control transfected 293 cells and subtracted from proliferation seen in SGP-containing supernatants after measuring $\left[{ }^{3} \mathrm{H}\right]$ thymidine incorporation.

$$
\text { d T enriched T tepleted }
$$
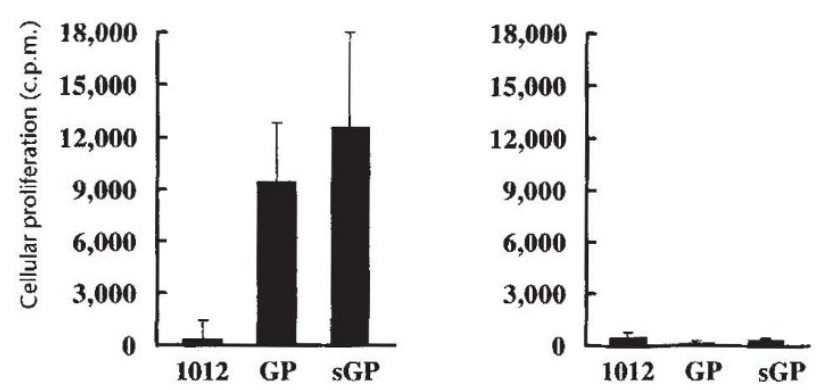

protein (NP), the secreted glycoprotein (sGP) or the membraneassociated glycoprotein (GP) were inserted. Alternative forms of GP were chosen because it had been postulated that the transmembrane protein contained a protein sequence motif also found in oncogenic retroviruses that might suppress immune responses $^{18-22}$. Expression of the relevant proteins was confirmed after transfection of the human renal epithelial cell line, 293, by immunoblotting with antiserum to these gene products (Fig. 1a). For NP, the expected full-length 104-kDa protein normally produced by the virus was seen, together with lower molecular weight species likely generated from truncated protein or degradation products described previously ${ }^{23}$. Similarly, expression of sGP and GP revealed a heterogeneous pattern whose sizes correlated with the expected products of cleavage or posttransiational carbohydrate modification ${ }^{3}$.

These plasmids were injected into mice to characterize their 
ability to induce humoral and cellular immune responses to the relevant viral proteins. Three injections, each with $50 \mu \mathrm{g}$ of plasmid DNA in saline $(100 \mu \mathrm{l})$, were performed at 2 -week intervals in $\mathrm{BALB} / \mathrm{c}$ female mice ( $6-8$ weeks old). Serum from immunized recipients were examined for antibody responses. An antibody response to the viral NP gene product was readily detectable (Fig. $1 \mathrm{~b}$ ), with titers of $\geq 1: 16,000$ by western blot analysis. The titer of antibody induced in response to injection with plasmids encoding the viral glycoproteins was lower. After immunization with GP, no antibody was detectable by western blotting, while immunization with sGP induced an antibody response (Fig. $1 b$ ). The more sensitive enzyme-linked immunosorbent assay (ELISA) $^{24,25}$ did allow detection of antibodies to both GP and SGP at titers of $1: 400$ and $1: 1200$, respectively. Cytolytic T-cell (CTL) responses to these viral proteins were analyzed next. Despite the substantial humoral immune response to NP, minimal CTL activity was detected against syngeneic cells expressing this viral protein (Fig. 2a). In contrast, genetic immunization with sGP, which elicited a weaker antibody response, induced a marked cytolytic T-cell response to cells expressing GP (Fig. 2b). Immunization with the GP plasmid also induced a significant CTL response to GP (Fig. 2c). These data suggested that both the secreted and transmembrane forms of the protein could be processed for antigen presentation and that the transmembrane form was a target for recognition by these cytolytic $\mathrm{T}$ cells. Finally, antigen-specific T-cell proliferation to sGP was also observed in mice injected with GP and sGP but not with control plasmid (Fig. $2 d$ ).

The ability of antibodies detected in mouse serum after immunization to neutralize virus was tested in an in vitro infection assay ${ }^{13}$. In no case was neutralization of infectivity observed, even at titers of 1:10 (data not shown), despite the documented presence of antibody after NP and sGP immunization by western blot analysis. Infectivity in vitro was thus not inhibited by Ebola-specific antibodies.

Protection of guinea pigs against lethal viral challenge To determine whether the in vivo immune responses could protect against viral infection, virus was adapted to grow in guinea pigs. This species is not well-suited to analysis of immune function; however, infection in adult mice has not been successful. Moreover, infection in guinea pigs, used originally to propagate virus from infected humans, is a wellestablished animal model for the human disease. Infection gives rise to a syndrome of hemorrhagic fever with levels of virus, organ pathology, and infection of reticuloendothelial and mononuclear cells comparable to those in humans ${ }^{26}$.

Two groups of immunized guinea pigs were studied. Animals were injected intramuscularly with the relevant expression vector plasmids, and the response to infection in groups immunized with sGP, GP, NP or control plasmids was observed. In the first group, animals were challenged within 2 months after the initial immunization, at which time the antibody titers were high, ranging from $1: 1,600$ to
$>1: 25,000$ (Table $1 a$ ). In these animals, nearly complete protection from lethal challenge was observed in GP $(6 / 6)$, sGP $(5 / 6)$ and NP (4/4) immunized subjects, in contrast to controls $(0 / 6)$. In a second group, guinea pigs were challenged 4 months after the initial immunization (Table $1 b$ ). As in the first group, all animals immunized with the control vector succumbed to infection within a week after virus challenge $(n=4)$. In this group, antibody titers were lower, and three of the four guinea pigs immu-

Table 1 Protection against lethal challenge with Ebola virus after DNA immunization correlates with antibody titer in guinea pigs

\begin{tabular}{|c|c|c|c|c|c|}
\hline \multicolumn{6}{|c|}{ a, Group I } \\
\hline Plasmid & Subject & ELISA (Pre) & ELISA (Post) & Viral Ag & Survival \\
\hline$G P$ & 1 & $>1: 25,600$ & $1: 12,800$ & - & Yes \\
\hline GP & 2 & $>1: 25,600$ & $1: 25,600$ & - & Yes \\
\hline GP & 3 & $>1: 25,600$ & $1: 25,600$ & - & Yes \\
\hline GP & 4 & $1: 25,600$ & $1: 6,400$ & - & Yes \\
\hline$G P$ & 5 & $1: 25,600$ & $1: 12,800$ & - & Yes \\
\hline$G P$ & 6 & $1: 25,600$ & $1: 25,600$ & - & Yes \\
\hline SCP & 1 & $1: 12,800$ & $1: 25,600$ & - & Yes \\
\hline SCP & 2 & $1: 6,400$ & $1: 25,600$ & - & Yes \\
\hline SCP & 3 & $1: 6,400$ & $1: 25,600$ & - & Yes \\
\hline SGP & 4 & $1: 25,600$ & $1: 25,600$ & - & Yes \\
\hline SGP & 5 & $>1: 25,600$ & $1: 12,800$ & - & Yes \\
\hline SGP & 6 & $1: 1,600$ & Negative & + & No \\
\hline NP & 1 & $1: 12,800$ & $>1: 25,600$ & - & Yes \\
\hline$N P$ & 2 & $>1: 25,600$ & $1: 25,600$ & - & Yes \\
\hline NP & 3 & $1: 12,800$ & $1: 12,800$ & - & Yes \\
\hline NP & 4 & $1: 25,600$ & $1: 25,600$ & - & Yes \\
\hline Vector alone & 1 & Negative & Negative & + & No \\
\hline Vector alone & 2 & Negative & ND & + & No \\
\hline Vector alone & 3 & Negative & Negative & + & No \\
\hline Vector alone & 4 & Negative & Negative & + & No \\
\hline Vector alone & 5 & Negative & ND & + & No \\
\hline Vector alone & 6 & Negative & ND & + & No \\
\hline \multicolumn{6}{|c|}{ b, Group II } \\
\hline Plasmid & Subject & ELISA(Pre) & ELISA(Post) & Viral Ag & Survival \\
\hline$G P$ & 1 & $1: 2,560$ & ND & $+/ f$ & No \\
\hline GP & 2 & $1: 5,120$ & $1: 10,240$ & - & Yes \\
\hline GP & 3 & $1: 10,240$ & $1: 10,240$ & - & Yes \\
\hline$G P$ & 4 & $1: 1,280$ & ND & $+/ f$ & No \\
\hline$G P$ & 5 & $1: 5,120$ & $1: 20,480$ & - & Yes (ill) \\
\hline SGP & 1 & $1: 2,560$ & ND & + & No \\
\hline SGP & 2 & $1: 10,240$ & $1: 5,120$ & $+/ f$ & Yes \\
\hline SGP & 3 & $1: 10,240$ & $1: 81,920$ & - & Yes \\
\hline SGP & 4 & $1: 2,560$ & $1: 5,120$ & - & Yes \\
\hline SGP & 5 & $1: 640$ & ND & + & No \\
\hline NP & 1 & ND & ND & + & No \\
\hline NP & 2 & ND & ND & + & No \\
\hline NP & 3 & ND & ND & + & No \\
\hline NP & 4 & ND & Negative & + & Yes (ill)* \\
\hline Vector alone & 1 & Negative & ND & + & No \\
\hline Vector alone & 2 & Negative & ND & + & No \\
\hline Vector alone & 3 & Negative & ND & + & No \\
\hline Vector alone & 4 & Negative & ND & + & No \\
\hline
\end{tabular}

Guinea pigs in group | were immunized on days $1,14,28,42$ and challenged on day 62 and those in group II on days $1,14,42$ and 112 and challenged on day 122 . Ag, antigen; ND, not determined. ELISA determinations were made before viral challenge (pre) or at least 7 days after (post) infection, respectively. Viral Ag denotes the presence of virus determined by immunohistochemistry ${ }^{30}$ performed on spleen, liver, lung, kidney and heart tissues; the +symbol denotes widespread systemic involvement of the mononuclear phagocyte system and to a lesser extent endothelial and parenchymal celis; $+/ f$ indicates focal involvement (seen in the spleen of SGP 2, the liver and spleen of GP 1, and the lung of GP 4) where rare sites of anti-Ebola antibody staining were detected.; - denotes no Ebola virus antigen detected in tissues

*The surviving NP immunized animal (4) was found to have significant levels of virus in major organs by immunohistochemistry, and more than 5 logs of virus was detected in the serum and spleen, in contrast to GP and SGP animals in which no virus was detected. 


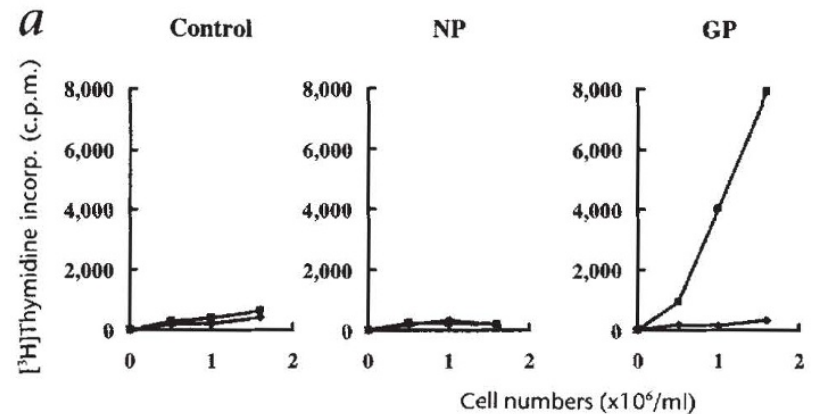

C

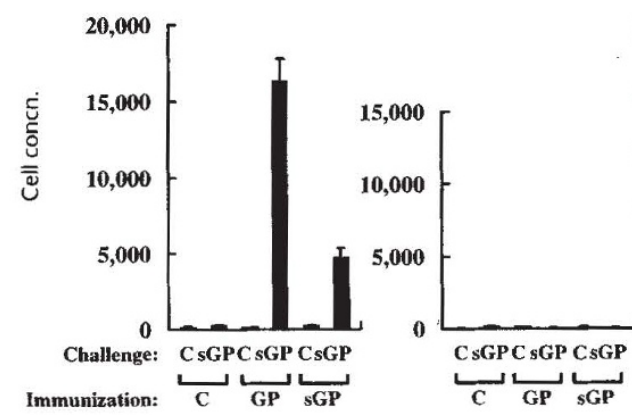
Spleen

T depleted

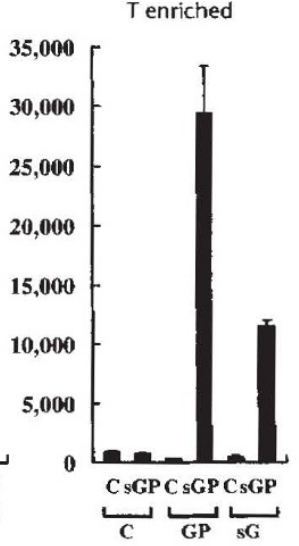

sGP

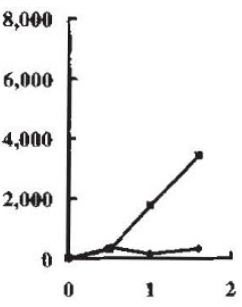

$b$

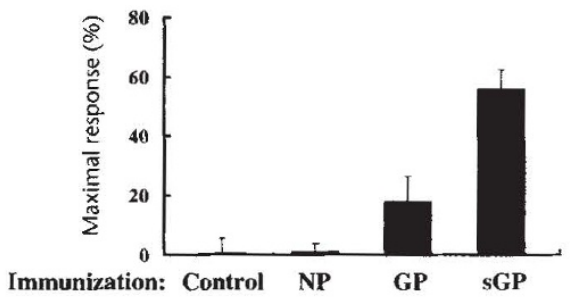

Fig. 3 Immunization with SGP or GP expression plasmids induces T-cell responses to SGP in guinea pigs. Cell-mediated immunity in guinea pigs was analyzed by performing assays to detect cell proliferation to control, $\mathrm{NP}$, or GP antigen (a) or T-cell growth factor production in response to the indicated antigens. The culture supernatants containing these antigens were prepared as previously described ${ }^{36,37}$ and included at a final concentration of $10 \%(\mathrm{vol} / \mathrm{vol})$. In $\boldsymbol{a}$, cell numbers refer to the concentration of spleen cells per milliliter in the $\left[{ }^{3} \mathrm{H}\right]$ thymidine proliferation assay. $\boldsymbol{b}$, Supernatants from $a$, harvested at the time of the peak proliferative response to SCP, were incubated with primary guinea pig $T$ cells maintained in $200 \mathrm{U} / \mathrm{ml}$ of human iL-2. The percent maximal response refers to the magnitude of stimulation in response to the indicated stimuli relative to supernatants from 24-h concanavalin A-stimulated cells $(2 \mu \mathrm{g} / \mathrm{ml})$. c, T lymphocytes are required for the proliferative response to $5 \mathrm{GP}$, performed as described in Methods at a cell concentration of $10^{6} / \mathrm{ml}$. nized with NP succumbed to infection, with the single survivor appearing severely ill after 1 week, in contrast to the protective response with NP at the earlier time point after immunization in group I. More effective protection was seen in animals immunized with vector expressing GP. Protection was noted in four of five animals challenged, with one survivor appearing weak but surviving the viral challenge. Similarly, three of the five animals immunized with sGP showed no ill effects following viral challenge. Protection in this group again correlated with the ability to sustain an effective immune response to GP or sGP. Together, all guinea pigs immunized with vectors expressing GP or sGP that had titers greater than 1:5120 were resistant to infection (11/11) compared with $0 / 10$ controls $(P=0$, by Fisher's exact test). Twelve of 14 animals with antibody titers $\geq 2560$ survived viral challenge compared with controls $(P=0.00003$, by Fisher's exact test).

\section{Immunopathologic analysis of guinea pig response}

As did immunized mice, guinea pigs injected with GP or sGP plasmids were able to generate cell-mediated immune responses to the viral glycoprotein in addition to the antibody response. These responses were antigen-specific and $\mathrm{T}$ cell-dependent, as detected in SGP antigen-dependent spleen cell proliferation and T-cell growth factor assays (Fig. 3, $a-c$ ). Thus, the ability to generate and sustain significant cellular immune responses in vivo correlated with protection from infection. Though antibody titer correlated with protection, cell-mediated immunity appeared necessary for protection, because passive transfer of antibody to GP does not confer protection ${ }^{1,16}$ and antiserum from protected guinea pigs did not inhibit virus replication in vivo $(n=3)$ or at a 1:10 dilution in vitro (data not shown). Since the Hartley guinea pig to which the virus has been adapted for growth is outbred, cellular adoptive transfer studies could not be performed.
Pathologic analysis of animals challenged with virus revealed widespread tissue necrosis and dissemination of virus by immunohistochemistry, as is seen in human disease. Virus load correlated with susceptibility to infection with titers of $\geq 10^{5}$ in infected animals compared with undetectable levels in immunized survivors. In infected animals, the liver, lung and spleen showed evidence of significant viral antigen by immunohistochemistry (Fig. 4, Table 1), and both reticuloendothelial and mononuclear phagocytic involvement was observed.

Determination of antibody response in animals that survived virus challenge revealed increases in the immune response to viral proteins when initial titers were lower (Table 1). Less consistent increases in antibody titers were observed in the NP immunized animals. These data suggest that Ebola virus infection may stimulate immunity in survivors of a viral challenge when immune responses are not optimal.

\section{Discussion}

In this article, we report our analysis of the immune response to selected Ebola virus proteins after genetic immunization in mice and testing of their ability to protect against lethal infection in a susceptible animal model, the guinea pig. The immune analyses performed in different species suggest similar patterns of response, though the specific peptides that may be recognized by the immune system to confer protection in the guinea pig could differ from those for the mouse. Because the principles of major histocompatibility complex (MHC) antigen presentation and recognition apply broadly across species ${ }^{27-29}$, the finding that protection was observed in different members of an outbred strain and that similar immune responses were seen in different species is not unexpected and suggests that this approach may be applicable to humans.

Immunization with plasmids encoding distinct viral proteins 
Protected
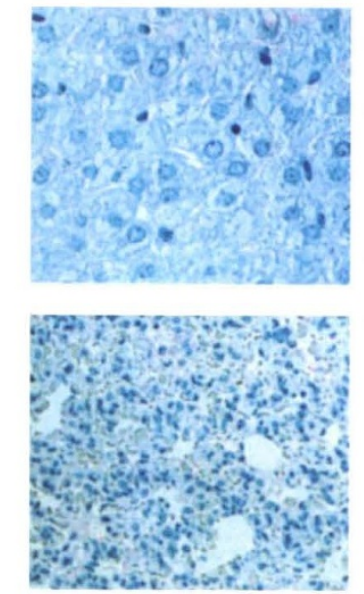

Spleen

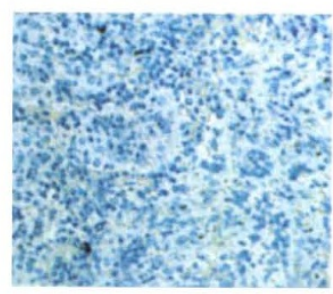

Infected
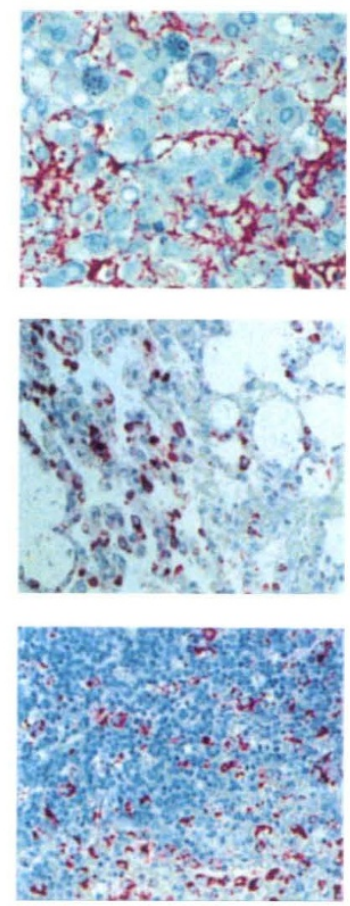

Fig. 4 Immunohistochemical analysis of Ebola virus antigens in liver, lung and spleen from representative protected (GP-animal 3) or infected (vectoranimal 2) guinea pigs. Magnification: liver, $\times 40$; lung, $\times 20$; spleen, $\times 20$.

induced different antibody and cytolytic T-cell responses. The broadest immune response was conferred by GP and sGP, which induced both cellular and humoral immunity to the membraneassociated GP. In guinea pigs challenged with doses of virus that are otherwise lethal, sGP provided nearly equivalent protection to GP, with no significant difference between these groups. The ability of vectors expressing GP to confer immunity may be explained by the generation of lower molecular weight degradation products (Fig. $1 b$ ), which could provide sufficient protein for antigen presentation to induce detectable, cellular and humoral immune responses in guinea pigs.

Despite the fact that plasmid DNA injection has been shown to affect the immune response to different antigens in infectious and autoimmune diseases, the ability of individual gene products to protect against disease in vivo is not readily predictable. In particular, the rapid rates of Ebola virus replication and the poor immunogenicity of its proteins had previously rendered it resistant to immune interventions. Several attempts to confer protection with passive transfer of immunoglobulin were unsuccessful ${ }^{1,16}$, in agreement with the finding here that antiserum from protected animals fails to neutralize virus replication in vitro. Previous studies using formalin-fixed virus or purified viral proteins for immunization have also not proven effective ${ }^{1,15}$.

It is likely that traditional immunization approaches using protein antigens, vaccinia virus or inactivated virus do not allow for appropriate uptake and presentation of viral antigens by dendritic or other antigen-presenting cells to induce protective immune responses. It has been shown recently that the genetic immunization leads to production of recombinant protein(s) in muscle, which are delivered to bone marrow-derived antigenpresenting cells $\mathrm{s}^{30-32}$. Synthesis of Ebola glycoprotein after gene transfer apparently allows more efficient processing and presentation and the generation of immune responses not seen with virus or with viral vectors. GP is a large molecule that contains both T- and B-cell epitopes. Although antibody levels provide a surrogate marker of protection, the fact that passive transfer of antibody did not confer protection implies that immunoglobulin switching and synthesis is reflective of the T-helper response to GP. Genetic immunization stimulates T-helper cells to generate both CTL and B-cell antibody responses to the virus. Although antibody production confirms effective immunization, a productive T-cell response, involving Th1 cell stimulation as shown by the T-cell proliferation and CTL assays (Fig. 3), is likely needed for effective immunity. Taken together, these studies suggest that transcription and translation of viral genes in host cells by genetic immunization induces alternative, more effective, processing and antigen presentation, which better stimulates immunity to Ebola virus. Because there are as yet no effective antiviral agents, the ability to generate protective immunity by vaccination may prove useful in selected high-risk populations, particularly in regions of ongoing outbreaks, and among medical and laboratory personnel exposed to the virus. Although it remains important to identify agents for treating acute infection, genetic immunization may help to limit the spread of this highly lethal, infectious disease.

\section{Methods}

Plasmids. Plasmids containing the GP, SGP or NP CDNAs (ref. 22) were used to subclone the relevant inserts into CMV expression vectors, which utilized the bovine growth hormone polyadenylation sequence ${ }^{17}$. Briefly, for GP, plasmid pGEM-3Zf(-)-GP was digested with EcoRI, treated with the Klenow fragment of Escherichia coli DNA polymerase, and digested with BamHI. The GP fragment was then inserted into the pCMV expression vector plasmid digested with Bg/ll Klenow fragment and BamHI. For SGP, the plasmid pCRII-sGP was digested with ECORI and treated with Klenow enzyme, and the resulting fragment was inserted into the BamHI/Bg/lI CMV plasmid, which had been incubated with Klenow fragment and calf intestinal phosphatase (CIP), and phenol chloroform extracted. For the NP expression vector, plasmid pSP64-NP2 (ref. 23) was digested with EcoRI/BamHI, and treated with Klenow enzyme. The NP insert was cloned into CMV treated with BamHI, Klenow enzyme, followed by heat inactivation and Bglll digestion.

Transient transfection and western blot analysis. Expression vectors encoding the viral gene products under regulation of the CMV immediate-early region 1 enhancer and promoter were prepared and transfected into 293 cells as previously described ${ }^{17,34}$. Cell extracts were prepared and analyzed by western blot analysis for NP or GP by using relevant rabbit antiserum and a secondary antibody, horseradish peroxidase-conjugated donkey anti-rabbit IgG, at a dilution of 1:5000. Incubation with primary antibody was for $30 \mathrm{~min}$ at room temperature, and for $30 \mathrm{~min}$ at room temperature with secondary antibody. Immunocomplexes were then detected by chemiluminescence using super signal substrate reagents (Pierce, Rockford, IL) according to manufacturer's instructions.

Antiserum samples from mice were tested at a dilution of 1:500 (NP), $1: 50(\mathrm{GP})$ or $1: 50$ (SGP), respectively, and developed with a secondary antibody (sheep anti-mouse, 1:5000; Amersham Life Science, Little Chalfont, Buckinghamshire, UK).

Cell lines and transfectants. For stable transfectants, the relevant CDNAs were transfected into Renca or $\mathrm{CT} 26$ which was syngeneic to $\mathrm{BALB} / \mathrm{C}$ mice by using calcium phosphate and selected in 0.7 or $1 \mathrm{mg} / \mathrm{ml} \mathrm{G} 418$ for 2-6 weeks. Expression of GP, sGP or NP from these vectors in Renca or CT26 cells was also confirmed by western blot analysis (data not shown).

Cell proliferation assay. Spleen cells from male Hartley guinea pigs or $\mathrm{BALB} / \mathrm{C}$ female mice (8-10 weeks, Charles River Laboratories, Wilmington, 
$M A)$ immunized with the indicated plasmid expression vectors were incubated with SGP or vector control supernatants $(25 \% \mathrm{vol} / \mathrm{vol})$ from transfected 293 cells at the indicated cell concentrations. T-cell depletion was performed by using the CT5 monoclonal antibody ${ }^{33}$ (Biosource, Camarillo, CA) for guinea pigs or anti-Thy 1.2 antibody in the mouse by using immunomagnetic microbeads (Miltenyi Biotec, Inc., Auburn, CA).

Viral challenge in guinea pigs. Animals were immunized by injection of $100 \mu \mathrm{l}(0.5 \mathrm{mg} / \mathrm{ml})$ in each hind leg (two injections at each time point) with the indicated plasmid expression vectors. Animals were challenged by inoculation with a stock of Ebola virus (Zaire, 1976) that had been passaged once in Vero $\mathrm{E} 6$ cells and serially passaged by intraperitoneal injection of spleen homogenates in Hartley guinea pigs seven times. Immunized guinea pigs were injected intraperitoneally with $0.5 \mathrm{ml}$ of a 1:1000 dilution of spleen cell homogenate in Hanks' balanced salt solution 122 days after the initial plasmid DNA injection (1000 plaque-forming units). Survival was determined 10 days later at which times animals were killed for serologic and pathologic analysis. ELISA, enzyme-linked immunosorbent assay ${ }^{21,22}$ on infected cell supernatants and enriched viral extracts containing GP, SGP or NP were performed as previously described.

\section{Acknowledgments}

We thank Donna Gschwend for preparation of the manuscript, Nancy Barrett for preparation of figures, and S.G. Trappier and G.L. Reynolds for their heip in the guinea pig infection studies.

\section{RECEIVED 4 AUGUST; ACCEPTED 7 NOVEMBER 1997}

1. Peters, C.I., Sanchez, A., Rollin, P.E., Ksiazek, T.G. \& Murphy, F.A. Filoviridae: Marburg and Ebola viruses. in Fields Virology. (eds. Fields, B.N., Knipe, D.M.\& Howley, P.M.) 1161-1176 (Lippincott-Raven, Philadelphia, 1996).

2. Peters, C.J. et al. Filoviruses as emerging pathogenesis. Semin. Virol. 5, 147-154 (1994).

3. Sanchez, A., Trappier, S.G., Mahy, B.W.J., Peters, C.J. \& Nichol, S.T. The virion glycoproteins of Ebola viruses are encoded in two reading frames and are expressed through transcriptional editing. Proc. Natl. Acad. Sci. USA 93, 3602-3607 (1996)

4. Wolff, I.A. et al. Direct gene transfer into mouse muscle in vivo. Science 247, $1465-1468$ (1990).

5. Tang, D.C., DeVit, M. \& lohnston, S.A. Genetic immunization is a simple method for eliciting an immune response. Nature 356, 152-154 (1992).

6. Ulmer, J.B. et al. Heterologous protection against influenza by injection of DNA encoding a viral protein. Science 259, 1745-1749 (1993).

7. Raz, E. et al. Intradermal gene immunization: the possible role of DNA uptake in the induction of cellular immunity to viruses. Proc. Natl. Acad. Sci. USA 91, 9519-9523 (1994).

8. Doolan, D.L. et al. Circumventing genetic restriction of protection against malaria with muitigene DNA immunization: $\mathrm{CD} 8^{+}$cell-, interferon gamma-, and nitric oxide-dependent immunity. J. Exp. Med. 183, 1739-1746 (1996).

9. Sedegah, M., Hedstrom, R., Hobart, P. \& Hoffman, S.L. Protection against malaria by immunization with plasmid DNA encoding circumsporozoite protein. Proc. Not\}. Acad. Sci. USA 91, 9866-9870 (1994).

10. Tascon, R.C. et al. Vaccination against tuberculosis by DNA injection. Nature Med. 2, 888-892 (1996).

11. Raz, E. et al. Systemic immunological effects of cytokine genes injected into skeletal muscle. Proc. Natl. Acad. Sci. USA 90, 4523-4527 (1993).

12. Waisman, A. et al. Suppressive vaccination with DNA encoding a variable region gene of the T-cell receptor prevents autoimmune encephalomyelitis and activates Th2 immunity. Nature Med. 2, 899-905 (1996).
13. McCormick, J.B., Bauer, S.P., Elliott, L.H., Webb, P.A. \& Johnson, K.M. Biological differences between strains of Ebola virus from Zaire and Sudan. 1. Infect. Dis. $147,264-267$ (1983).

14. Border, W.A. \& Noble, N.A. Targeting TCF- $\beta$ for treatment of disease. Noture Med. 1, 1000-1001 (1995).

15. Clegg, J.C.S. \& Sanchez, A. Vaccines against arenaviruses and filoviruses. in New Generation Voccines. (eds. Levine, M.M., Woodrow, G.C., Kaper, I.B.\& Cobon, G.S.) 749-765 (Marcel Dekker, New York, 1997).

16. Jahrling, P.B. et al. Passive immunization of Ebola virus-infected cynomolgus monkeys with immunoglobulin from hyperimmune horses. Arch. Virol. Suppl. 11, 135-140 (1996).

17. Manthorpe, $M$. et al. Gene therapy by intramuscular injection of plasmid DNA: Studies on firefly luciferase gene expression in mice. Hum. Gene. Ther. 4, 419-431 (1993).

18. Burkreyev, A.A., Volchkov, V.E., Blinov, V.M. \& Netesov, S.V. The GP-protein of Marburg virus contains the region similar to the "immunosuppressive domain" of oncogenic retrovirus P15E proteins. FEBS. Lett. 323, 183-187 (1993).

19. Cianciolo, G.J., Copetand, T.J., Oroszlan, S. \& Snyderman, R. Inhibition of lymphocyte proliferation by a synthetic peptide homologous to retroviral envelope protein. Science 230, 453-455 (1985).

20. Harris, D.T., Cianciolo, C.J., Snyderman, R., Argov, S. \& Koren, H.R. Inhibition of human naturai killer cell activity by a synthetic peptide homologous to a conserved region in the retroviral protein, p15E. J. Immunol. 138, 889-894 (1987).

21. Volchkov, V.E., Blinov, V.M. \& Netesov, S.V. The envelope glycoprotein of Ebola virus contains an immunosuppressive-like domain similar to oncogenic retroviruses. FEBS. Lett. 305, 181-184 (1992).

22. Sanchez, A., Kiley, M.P., Holloway, B.P. \& Auperin, D.D. Sequence analysis of the Ebola virus genome: Organization, genetic elements, and comparison with the genome of Marburg virus. Virus. Res. 29, 215-240 (1993).

23. Sanchez, A., Kiley, M.P., Holloway, B.P., McCormick, J.B. \& Auperin, D.D. The nucleoprotein gene of Ebola virus: Cloning, sequencing, and in vitro expression. Virology 170, 81-91 (1989).

24. Ksiazek, T.C. Laboratory diagnosis of filovirus infections in nonhuman primates. Lab. Anim. 20, 34-46 (1991).

25. Ksiazek, T.G. et al. Enzyme immunosorbent assay for Ebola virus antigens in tissues of infected primates. f. Clin. Microbiol. 30, 947-950(1992).

26. Bowen, E.T.W. et al. Viral haemorrhagic fever in southern Sudan and northern Zaire. Lancet 1, 571-573 (1977).

27. Monaco, J.J. A molecular model of MHC class-1-restricted antigen processing. immunol. Today 13, 173-179 (1992).

28. Jorgensen, J.L.., Reay, P.A., Ehrich, E.W. \& Davis, M.M. Molecular components of Tcell recognition. Annu. Rev. immunol. 10, 835-873 (1992).

29. Zinkernagel, R.M. \& Doherty, P.C. The discovery of MHC restriction. Immunol. Today 18, 14-17 (1997).

30. Corr, M., Lee, D.J., Carson, D.A. \& Tighe, H. Gene vaccination with naked plasmid DNA: Mechanism of CTL priming. I. Exp. Med. 184, 1555-1560 (1996).

31. Doe, B., Selby, M., Barnett, S., Baenziger, J. \& Walker, C.M. Induction of cytotoxic T lymphocytes by intramuscular immunization with plasmid DNA is facilitated by bone marrow-derived cells. Proc. Natl. Acad. Sci. USA 93, 8578-8583 (1996).

32. iwasaki, A., Torres, C.A.T., Ohashi, P.S., Robinson, H.L. \& Barber, B.H. The dominant role of bone marrow-derived cells in CTL induction following plasmid DNA immunization at different sites. I. Immunol. 159, 11-14 (1997).

33. Tan, B.T.G. et al. Production of monoclonal antibodies defining guinea pig T-cell surface markers and a strain 13 la-like antigen: The value of immunohistological screening. Hybridoma 4, 115-124 (1985).

34. Sambrook, I., Fritch, E.F. \& Maniatis, T. Molecular Cloning: A Laboratory Manual (Cold Spring Laboratory Harbor Press, Cold Spring Harbor, NY, 1994).

35. Ohno, T. et af. Combination gene transfer to potentiate tumor regression. Gene. Ther. 4, 361-366 (1997).

36. Bottomly, K., Davis, L.S. \& Lipsky, P.E. Measurement of human and murine interleukin 2 and interleukin 4. in Current Protocols in Immunology. (eds. Coligan, J.E., Kruisbeek, A.M., Margulies, D.H., Shevach, E.M. \& Strober, W.) 6.3.1-6.3.12 (Wiley \& Sons, New York, 1992).

37. Arai, H., Chan, S.Y., Bishop, D.K. \& Nabel, G.J. Inhibition of the alloantibody response by CD9S ligand. Nature Med. 3, 843-848 (1997). 